\title{
Fault Line Selection Method Based on Improved HHT Small Current Ground
}

\author{
Xu Jianjun ${ }^{1}$, Gao Xuehan ${ }^{2}$ \\ ${ }^{1,2}$ School of Northeast Petroleum University, Daqing, 163318, China
}

\begin{abstract}
In the analysis of nonlinear, non-stationary signal is better than the traditional wavelet signal analysis method, commonly used in this kind of signal singular value point detection. Empirical mode decomposition (EMD) is the core of the analysis method, but EMD in the original HHT method exists end effect in decomposition process, the decomposition of intrinsic mode functions IMF serious distort, greatly affected the accuracy of the fault line selection, by extension on the endpoint can inhibit the effect of the end point. In this paper, we compare the different endpoint extension methods, and use the method of endpoint extension based on adaptive waveform matching to improve the algorithm. MATLAB simulation results show that the proposed method is effective and accurate in the fault location of small current grounding system.
\end{abstract}

Keywords: small current grounding system; Hilbert-Huang transform; end effect; fault line selection

\section{Introduction}

Low and medium voltage power network is mainly used in small current grounding system, there are neutral grounding, arc suppression coil grounding and high impedance grounding in three ways. The fault of small current grounding system is mainly single-phase ground fault. After single-phase grounding, the non fault phase voltage rise, but the line voltage is still symmetrical, so the system can continue to run $1 \sim 2 \mathrm{~h}$. However, if the long-term operation, it may cause the weak link of insulation breakdown, further expansion of the phase to phase short circuit fault, the power system caused a significant loss. For using the harmonic components of the line selection method, harmonics in the signal is a smaller proportion, it is difficult to separate and extract, coupled with the load of harmonic interference. It makes the device based on the principle of harmonic in the practical application of a miscarriage of justice. Wavelet analysis have variable frequency window, the changing characteristics of transient signal can be analyzed very well, but because of the method needs to set the wavelet function and decomposition scale. There is a certain degree of variability and transient process of short duration. The signal is difficult to capture and extraction. In the practical application it is difficult to achieve accurate fault line selection; fault line selection device for fault line selection method mostly based on the analysis of fault steady signal, due to interference by outside interference factors resulting in grounding the steady zero sequence current is very small, the accuracy of the line selection based on the amplitude comparison of protection reduced. The single-phase grounding fault characteristics on neutral ground via arc suppression coil grounding system are analyzed in theory in this paper. The waveform matching endpoint extension method to improve the extension first and after the interception of signals. Through the simulation validate the method the end effect of inhibiting effect. The model of small current grounding system is established by MATLAB. Using this model, the application effect of the improved pre - and post - Method in the fault line selection of single phase to ground fault is compared and analyzed. MATLAB simulation results show that this method has high accuracy and reliability.

\section{HHT Theory and End Effect}

\subsection{Instantaneous Frequency and Intrinsic mode Function (IMF)}

If the instantaneous frequency of a signal has a physical meaning, it must be satisfied that it is symmetrical, the local mean is zero, and the zero crossing point and the number of the extreme points are equal. Instantaneous frequency is the earliest respectively defined by Carson and Gabor ,the later Ville unified the two different definitions. The instantaneous frequency of any continuous signal $X(T)$ is defined as: the derivative of the phase of the analytic signal $\mathrm{Z}(\mathrm{t})$, that is: firstly, the $\mathrm{X}(\mathrm{T})$ is obtained after Hilbert-Huang transform.

Its Hilbert-Huang inverse transform is

$$
\mathrm{Y}(\mathrm{t})=\frac{1}{\pi} \int_{-\infty}^{+\infty} \frac{\mathrm{x}(\tau)}{\mathrm{t}-\tau} \mathrm{d} \tau
$$

$$
X(t)=\frac{1}{\pi} \int_{-\infty}^{+\infty} \frac{Y(\tau)}{t-\tau} d \tau
$$

$\mathrm{X}(\tau)$ and $\mathrm{Y}(\tau)$ can form a complex conjugate pair, and get the analytic signal:

$$
\mathrm{Z}(\mathrm{t})=\mathrm{X}(\mathrm{t})+\mathrm{jY}(\mathrm{t})=\mathrm{a}(\mathrm{t}) \mathrm{e}^{\mathrm{j} \theta(\mathrm{t})}
$$




$$
\begin{gathered}
a(t)=\sqrt{\left[\mathrm{X}^{2}(\mathrm{t})+\mathrm{Y}^{2}(\mathrm{t})\right]} \\
\theta(\mathrm{t})=\arctan \frac{\mathrm{Y}(\mathrm{t})}{\mathrm{X}(\mathrm{t})}
\end{gathered}
$$

That $\mathrm{a}(\mathrm{t})$ is the instantaneous amplitude, the $\theta(\mathrm{t})$ is the phase angle, the instantaneous frequency calculation formula is:

$$
f(t)=\frac{1}{2 \pi} \frac{d \theta(t)}{d t}
$$

From the formula, instantaneous frequency at any one time only corresponding to a frequency, when encountered many components in either a moment, the signal exists many frequency components of the complex signal. We should be the signal which was decomposed into multiple single component signal, in order to reflect the physical meaning of the instantaneous frequency of a signal. In order to obtain the instantaneous frequency of physical meaning, the concept of intrinsic mode function (IMF) is proposed by Norden. E. Huang and so on. The concept of intrinsic mode function is one of the major innovations of the empirical mode decomposition (EMD) method.

The intrinsic mode function (IMF) is defined as a signal that satisfies the following two conditions:

1. The sum of local maximum and local minimum values must be equal to or at least 1 of the number of zero crossing, that is to say, an extreme value must be immediately followed by a zero crossing.

2. At any point on the signal, the envelope and the envelope which are determined by the local maximum points are zero, that is, the signal is about the local symmetry of the time axis.

Such as a simple harmonic component, the intrinsic mode function (IMF) use time-varying amplitude and frequency, instead of a constant amplitude and frequency. Due to the intrinsic mode function (IMF) component has a physical meaning of instantaneous frequency, which makes it possible to analyze the time frequency based on Hilbert transform.

\subsection{Empirical Mode Decomposition (EMD)}

In order to make use of the Hilbert transform to analyze the nonlinear and non-stationary signals, the signal decomposition cost intrinsic mode function (IMF) is first used, and the empirical mode decomposition (EMD) is developed. Empirical mode decomposition (EMD) is through the layers of screening to obtain a series of intrinsic mode function (IMF), and the frequency of the intrinsic mode function (IMF) more and more low, which is the first IMF frequency was the highest, and finally get the IMF frequency minimum. Therefore, its essence is a filter bank.

Empirical mode decomposition (EMD) decomposes the signal into a series of intrinsic mode function (IMF) of the combination. Empirical mode decomposition (EMD) is an intrinsic mode function (IMF), which is gradually found by the repeated screening process. Signal x ( $t$ ) empirical mode decomposition (EMD) process can be summarized as follows:

Set the input signal $\mathrm{x}(\mathrm{t})$, then all of its maximum value, minimum value each with a curve together, so that the two curves between contains all of the signal data, as the upper envelope $U(t)$ and the lower envelope $V$ $(t)$ of $\mathrm{X}(\mathrm{t}), \mathrm{m}(\mathrm{t})$ as the mean value of upper and lower envelope. Order,

$$
\begin{aligned}
& \mathrm{m}(\mathrm{t})=\frac{[\mathrm{u}(\mathrm{t})+\mathrm{v}(\mathrm{t})]}{2} \\
& \mathrm{~h}_{1}(\mathrm{t})=\mathrm{x}(\mathrm{t})-\mathrm{m}(\mathrm{t})
\end{aligned}
$$

$h_{1}(t)$ can be regarded as a new $x(t)$, repeat the process until the resulting $h_{k}(t)$ to meet certain conditions $h_{k}(t)$ is small enough. At this point the decomposition is obtained for the first IMF1 component $\mathrm{c}_{1}(\mathrm{t})$ and the signal remaining part $\mathrm{r}_{1}(\mathrm{t})$. That is,

$$
\begin{gathered}
c_{1}(t)=h_{k}(t) \\
r_{1}(t)=x(t)-c(t)
\end{gathered}
$$

The remaining part of the signal R1 (T) continues to carry out EMD decomposition. Until the remaining part of the resulting signal is a single signal or its value is less than the set value, the decomposition is completed. Finally get all the IMF and the residual amount. The original $x(T)$ is the sum of all IMF and residual amounts.

\subsection{HHT Endpoint Effect Analysis}

$$
x(t)=\sum_{i=1}^{n} c_{i}+r_{n}
$$

EMD is a repeated process, an EMD is based on previous EMD of the IMF, unceasingly along with the decomposition, the original signal end point error will gradually spread and affect every decomposition accuracy, resulting in distortion of all the IMF.

Inspection signal $x(t)$ 


$$
\begin{gathered}
x(t)=\cos (0.04 \pi t)+\sin (0.08 \pi t) \\
t \in[0,100]
\end{gathered}
$$

Through the original EMD Method is shown in the original signal $\mathrm{x}(\mathrm{T})$ decomposition of the income under the envelope as shown in the figure, at both ends signal appeared serious "end wing" phenomenon. This greatly affects the accuracy of the signal analysis. Therefore, this article has carried on the improvement to it.

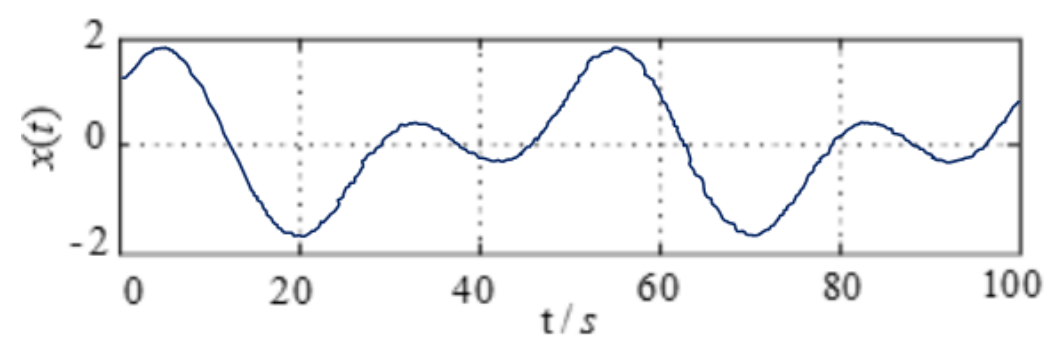

(a) Original Signal

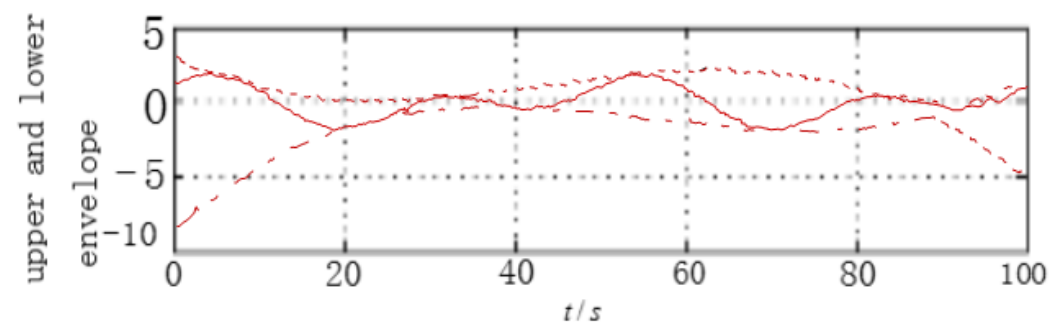

(b) Signal after EMD decomposition

Fig.1 Original signal and its up-down envelope

\subsection{Improvement of HHT Algorithm}

\section{Improvement and Application of HHT}

The end effect of EMD decomposition is the main factor affecting the accuracy of the method, and it is necessary to extend the end point of the signal sequence so as to get the accurate fault information. Existing some endpoint extension methods are: 1) directly to the endpoint as the extreme points of the endpoint extension, that is currently to be analyzed data endpoint as extreme points, this method most simple, but the effect is not ideal; (2) neural network endpoint extension method, slow and for different signals, suitable for neural network model is also different; (3) the mirror extending extension method for short data sequences treatment effect is poor.

The proposed by Zhang Kang and many people. Application to the decomposition of adaptive waveform matching continuation method, which is from inside the original signal to find the appropriate trend signal waveform of the signal extension and maximize maintain signal of the internal changes in the trend. The data signal extension includes two endpoints. The following is the extension as an example to illustrate the method. Set the original signal for $x(t), a_{j}, b_{j}(j=1,2,3, \ldots)$ respectively the signal $x(t)$ maxima and minima. The corresponding time are the $t_{a_{j}}$ and $t_{b_{j}}$. Set $x(1)$ as the left end point values of the $x(t) . x(1), a_{1}$ and $b_{1}$ three points form a triangle $S_{1}$. As shown in the figure, from the time interval $t_{a_{1}}-t_{b_{1}}$ of the waveform is called the characteristic waveform, and then along the signal $\mathrm{x}(\mathrm{t})$ search and the characteristic waveform is the most matching of the triangle waveform $x(j)-a_{j}-b_{j}$ that is $S_{2}$, defined as the matching waveform.

Specific steps are as follows:

(1) Find out the value $\mathrm{x}(\mathrm{j})$ of all the triangular waveform starting points except the characteristic waveform, and draw the corresponding time point according to the triangle similarity principle:

$$
t_{x(j)}=\frac{\left(t_{a_{1}} t_{b_{j}}-t_{b_{1}} t_{a_{j}}\right.}{\left(t_{a_{1}}-t_{b_{1}}\right)}
$$

(2) The matching error between all triangular waveform and characteristic waveform is calculated, and the error can be defined as:

$$
e(j)=\left|a_{j}-a_{1}\right|+\left|b_{j}-b_{1}\right|+|x(j)-x(1)|
$$

(3) Find the minimal matching error values mine(j), and set a threshold $\delta$, if mine $(\mathrm{j})<\delta$, mine (J) corresponding to the triangle waveform as the waveform matching, it will match the waveform data extension to the front of the original signal, if mine(j), jump to the next step. The threshold value can be adjusted according to the actual situation. The weaker threshold, the more match for the original signal. On the contrary, it is 
indicated that the intrinsic law of the original signal is weaker, or the boundary data is more abnormal.

(4) Directly set the extreme of the endpoints of the signal, respectively, and calculated the closest to the signal terminal, adjacent to the mean of $\mathrm{M}$ maximum points $\mathrm{A}$ and the mean of $\mathrm{N}$ the minimum points $\mathrm{B}$. The signals are the maximum and minimum value of $\mathrm{x}(\mathrm{t})$, the specific $\mathrm{A}, \mathrm{B}$ value of the positive and negative can be based on the signal at the boundary of the jump condition.

(5) According to the same method, extension of the right side of the signal. The signal at both ends of the extension is decomposed, the decomposition of the component according to the original signal corresponding to the moment of interception, so as to obtain improved decomposition results.

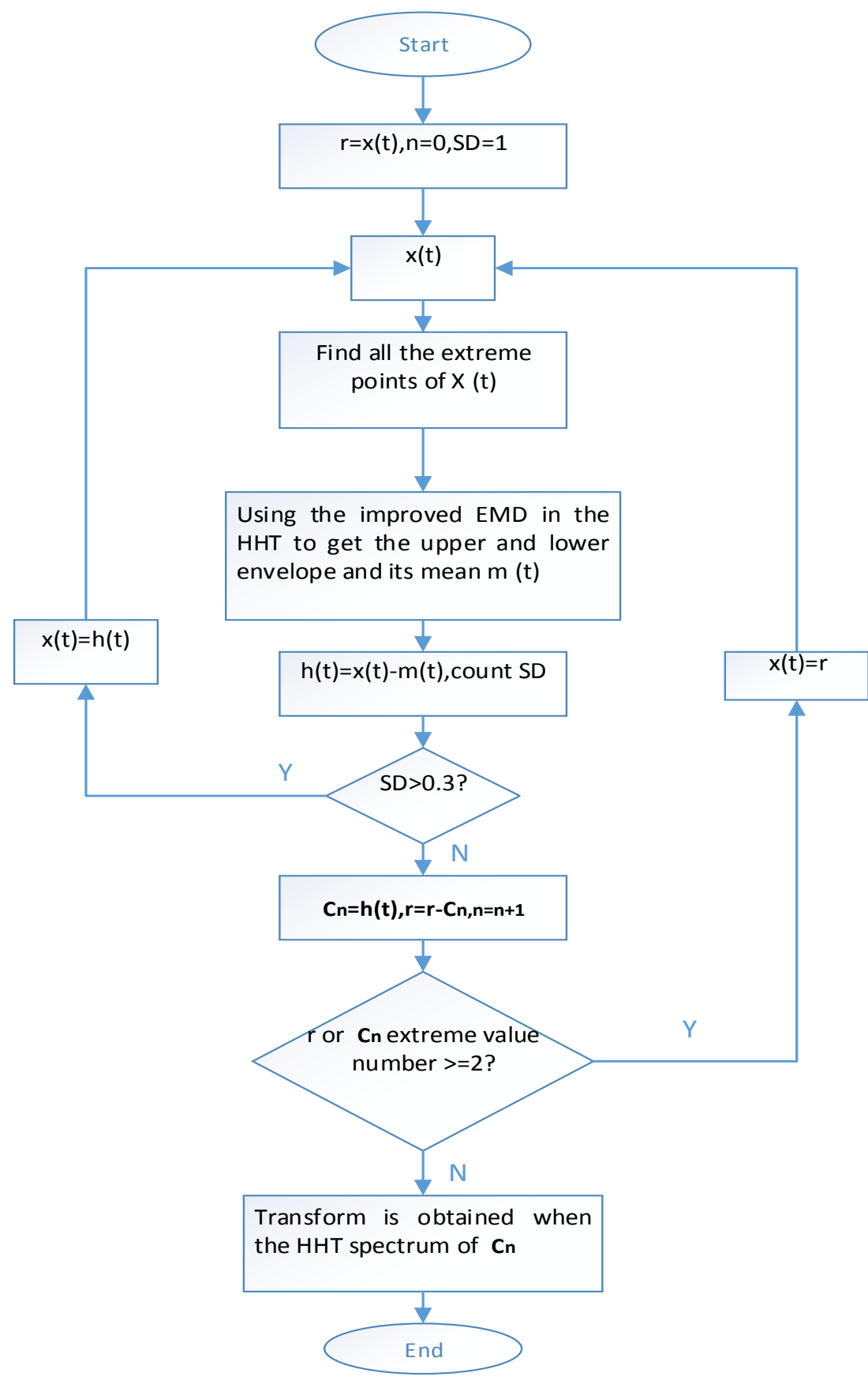

In Figure 1(a) of the given signal $\mathrm{x}(\mathrm{t})$ application improved HHT EMD decomposition, which income of the envelope line as shown in Figure 2. All the information is included in the envelope and at the end of the "flying wing" phenomenon, showing a smooth matching envelope waveforms, reflecting the effectiveness of adaptive waveform matching endpoint extension method. 


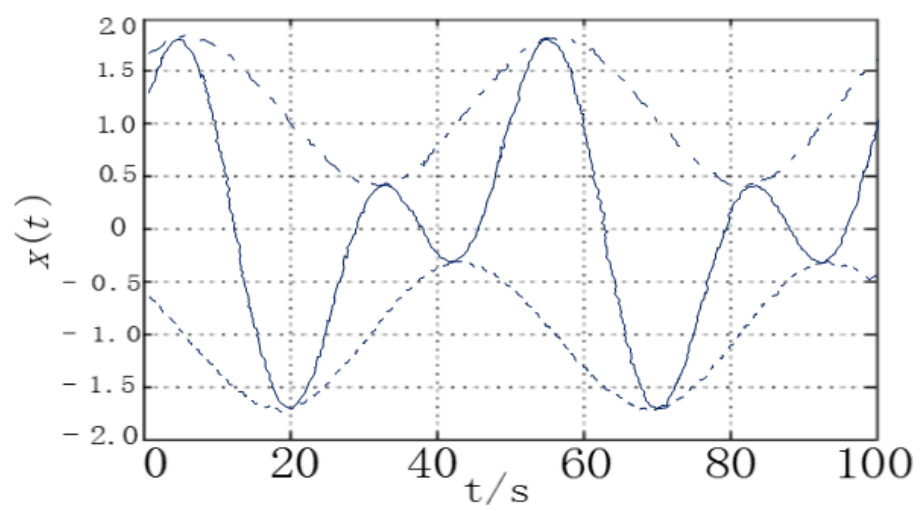

Fig.2 Up-down Envelope of Extended End Effect

\subsection{Fault line selection principle}

When a single phase to earth fault occurs in the small current grounding system, the zero sequence current occurs in the transmission line. And the polarity of the zero sequence current of the fault line and the sound circuit is opposite. Improved method in fault line selection, the main use of singularity detection method of fault signal in the mutation signal for precise position. The polarity phase of the fault is opposite to the criterion in the fault line and the non fault line, and the polarity of each line is judged. If the polarity of a line and other lines in the fault time are the opposite, it is determined that the line for the fault line, if the polarity is the same, then determine the bus fault.

Fault line selection procedures are as follows:

(1) By using the EMD method of HHT, the zero sequence current of each $1 / 2$ period is decomposed to obtain a series of IMF components, and the most high frequency IMF1.

(2) Comparison of the zero sequence current of each line IMF1 component in the zero sequence current mutation moment of the first order differential polarity (diff (IMF1)).

(3) If the zero sequence current of a circuit is the highest frequency IMF component of the first order differential polarity and other lines, the line is judged to be the fault line, otherwise, it is the fault of the bus.

\section{Simulation Verification}

Figure 3 for single-phase grounding fault of L3 line simulation model, the power supply is $35 \mathrm{~K} \mathrm{~V}$ transformer using $\Delta / \mathrm{YN}$ connection mode, the neutral point of the resonant $\mathrm{L}$ of arc suppression coil grounding, fire arc suppression coil compensation of $8 \%$, the ratio of $35 \mathrm{kv} / 10 \mathrm{kv}$. Line model using distributed parameter line model, bus connection 5 back to the overhead outlet line L1-L5, the length is: $12 \mathrm{~km} 、 18 \mathrm{~km} 、 20 \mathrm{~km} 、 15 \mathrm{~km}$ 、 $25 \mathrm{~km}$. Line parameters: $\mathrm{R} 1=0.45 \Omega / \mathrm{km}, \mathrm{L} 1=1.714 \mathrm{~m} \mathrm{H} / \mathrm{km}, \mathrm{C} 1=0.091 \mu \mathrm{F} / \mathrm{km} ; \mathrm{R} 0=0.7 \Omega / \mathrm{km}, \mathrm{L} 0=3$. $9065 \mathrm{~m} \mathrm{H} / \mathrm{km}, \mathrm{C} 0=0.038 \mu \mathrm{F} / \mathrm{km}$. Using MATLAB to simulate the network. The simulation time is $1 / 2 \mathrm{a}$ period of $002 \mathrm{~s}$ before and after the fault, the system has a single phase earth fault in L3 10km at $001 \mathrm{~s}$.

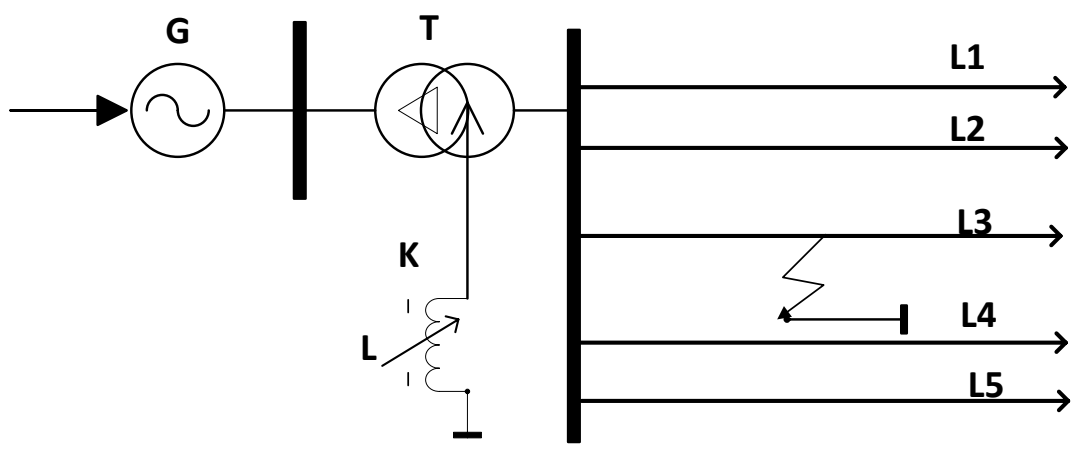

Fig.3 Non-solidly earthed system grid model

From Figure shows the original HHT method in the fault occurrence time of 0.01 not only the obvious phenomenon of "end of the wing, inward" pollution "signal, wave clutter, and is not easy to line selection. The improved HHT method of detection results as shown in Figure shown, before the failure without loss of signal, larger amplitude and inhibit the "end wing" phenomenon, waveform uniformity without interference. L3 and other lines of the opposite polarity at $0.01 \mathrm{~s}$, then determine the line 3 for the fault line, the line selection effect is more ideal. 


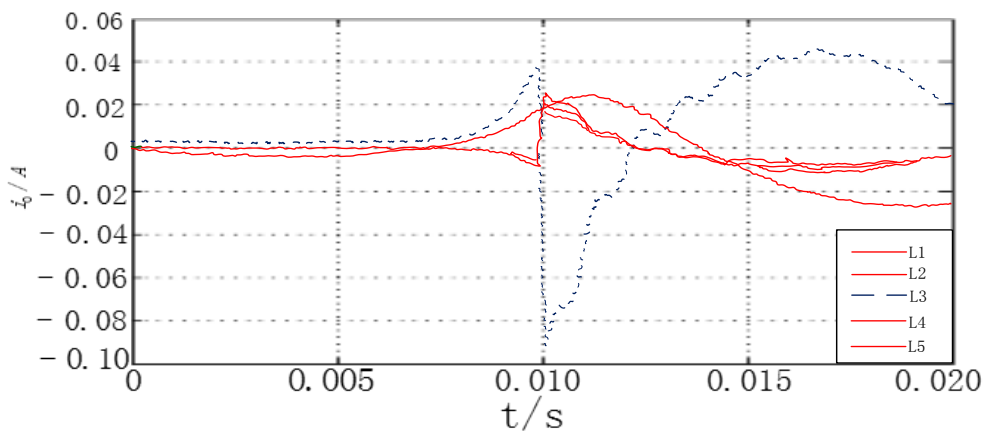

(a) Before Improvement

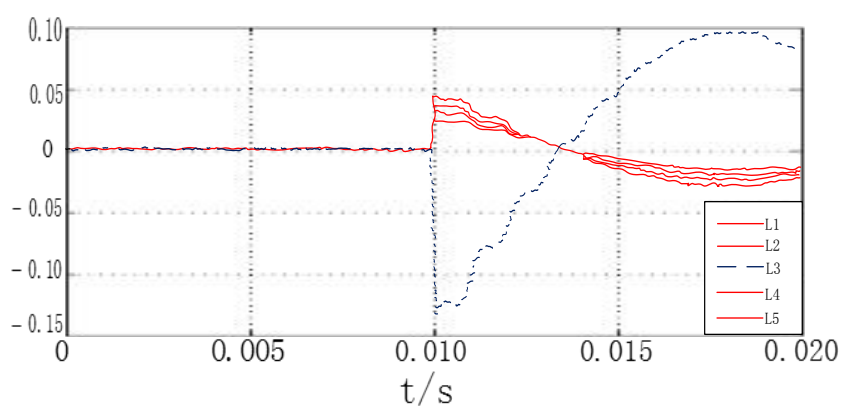

(b) After improvement

Fig.4 Original and Improved HHT Applied in test of Singularity

\section{Conclusion}

This chapter analyzes the principle of fault line selection based on improved, and introduces the specific steps to improve the line selection. The simulation model of small current grounding system is built by using software. The use of the original signal inside the sub waveform of the signal extension, so that the extension of the data to maintain the original signal change trends and laws, effectively inhibit the EMD decomposition of the end effect. Through the established simulation model, the improved pre and post methods are applied to the simulation of fault line selection, and the effect of the selection of the two methods is compared. Simulation results show that the improved not only effectively inhibited the decomposition process of end wing, but also improve the accuracy of fault line selection.

\section{References}

[1] Xu Qingshan. Power system fault diagnosis and fault restoration Beijing China Electric Power Press, 2007.

[2] Huang Daji and Zhao jinping. Practical implementation of the Hilbert-Huang transform algorithm. ACTA OCEANOLOGICA SINICA, 2003,25,1.

[3] Gabriel Rilling, Patrick Flandrin and Paulo Goncalves. On Empirical Mode Decomposition And Its Alglrithms. IEEE Sig. Proc. Lett, 2003.

[4] Huang S J, Hsieh C T. High Impedance Fault Detection Utilizing a Morlet Wavelet Transform Approach. IEEE Trans. on Power Delivery, 1999, 14(4):1401-1410.

[5] Thomas Baldwin, Frank Renovich. Fault Locating in Ungrounded and High Resistance Grounded Systems. IEEE Trans. on Industry Application, 2001,37(4):1152-11159.

[6] Panigrahi B.K, Mishra,S. Intelligent Classification of Power Quality Disturbances in a Distribution Network. TENCON 2005-2005 IEEE Region 10 Conference, Melbourne of Australia, 2005. 